MS8 Membranes and

membrane interacting proteins
Chairs: Han Remaut, Maike Bublitz

\section{MS8-01 Gating MscS: Structural basis of mechanosensation and the role of lipids in ion channel regulation}

Christos Pliotas ${ }^{1}, \mathrm{AC} \mathrm{Dahl}^{2}$, T Rasmussen ${ }^{3}, \mathrm{KR}^{2}$ Mahendran $^{4}$, TK Smith $^{1}$, P Marius ${ }^{1}$, J Gault ${ }^{4}$, A Rasmussen ${ }^{3}$, CV Robinson ${ }^{4}, \mathrm{H}$ Bayley $^{4}$, MS Sansom ${ }^{2}$, IR Booth ${ }^{3}$, JH Naismith ${ }^{1}$

1. Biomedical Sciences Research Complex, School of Chemistry, University of St Andrews, St Andrews, United Kingdom

2. Department of Biochemistry, University of Oxford, Oxford, United Kingdom

3. Institute of Medical Sciences, University of Aberdeen, Aberdeen, United Kingdom

4. Department of Chemistry, University of Oxford, Oxford, United Kingdom

email: cp39@st-andrews.ac.uk

Organisms use lipid bilayers impermeable to ions and polar molecules, to compartmentalize. The exchange of molecules with the outside world occurs in a controlled manner via ion channels, which are pore-forming proteins and respond to specific stimuli. Mechanosensitive ion channels in bacteria gate in response to changes in the lateral tension in the lipid bilayer and act as pressure safety valves, to prevent lysis during extreme osmotic shock.

Despite years of efforts, the gating models proposed, failed to accurately describe the structural transitions these systems undergo during gating ${ }^{1}$ and identify an effective molecular trigger. A combination of experimental methods, spanning from biochemistry (site-directed-spin-labeling) and biophysics (EPR spectroscopy, W-fluorescence, MD simulations and single molecule electrophysiology), to analytical chemistry (native and ES mass spectrometry) and structural biology (x-ray crystallography), were introduced to elucidate gating of the mechanosensitive channel $\mathrm{MscS}^{1,2}$.

Alkyl chains inside hydrophobic crevices were resolved in a new high-resolution x-ray MscS structure ${ }^{2}$. Native and electrospray mass spectrometry demonstrated that purified protein contained endogenous lipids transferred from the parental E. coli membrane. W-fluorescence quenching showed that exogenously added phospholipids exchange between bulk lipid bilayer and the TM hydrophobic pockets. MD simulations and structural analyses suggested that the volume of the pockets and the contained lipids decrease during channel opening, while externally added lyso-phospholipids (LPC) displace the endogenous lipids to trigger channel opening ${ }^{2}$.
We propose a new gating model by which the extent of lipid partitioning within transmembrane pockets determines the conformation of $\mathrm{MscS}$, a model with potential applicability to a wide range of membrane proteins.

\section{References}

1. Pliotas C, et al. (2012) PNAS 109(40) :E2675-2682

2. Pliotas C, et al. (2015) Nat Struct Mol Biol 22(12) :991-998

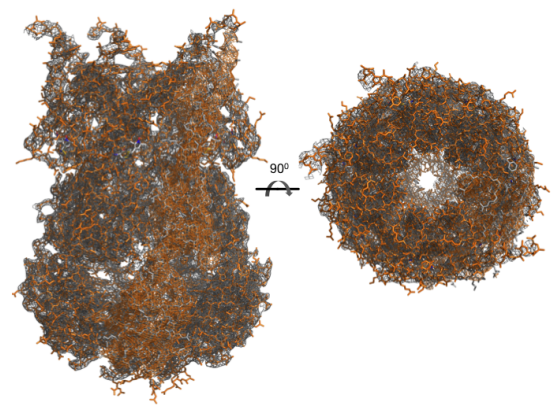

Figure 1. X-ray structure (side and top-pore views) of the MscS mechanosensitive ion channel (PDB 5AJI)

Keywords: mechanosensation, ion channels, mechanosensitive channels, MscS, lipids 